\title{
TRANSFORMACIÓN NEUROÉTICA DE LA RACIONALIDAD ECONÓMICA: IMPLICACIONES PARA TOMAR DECISIONES EN LAS EMPRESAS ${ }^{1}$
}

\author{
JOSÉ-FÉLIX LOZANO \\ Universidad Politécnica de Valencia
}

\begin{abstract}
RESUMEN: El ámbito de la economía y los negocios ha sido tradicionalmente un ámbito dominado por una concepción de racionalidad calculadora, estratégica y maximizadora del beneficio individual. Ese modelo de racionalidad tiene importantes limitaciones teóricas y graves consecuencias prácticas. En las últimas décadas los resultados de las investigaciones neuroéticas parecen abrir nuevas vías en la comprensión de la racionalidad práctica. Estas nuevas vías, que suponen una revalorización de la dimensión emocional e intuitiva en la capacidad cognitiva, tienen gran relevancia para la toma de decisiones en el ámbito de los negocios y las organizaciones.

El objetivo de este artículo es analizar las implicaciones de un concepto de racionalidad práctica dialógica-cordial para la toma de decisiones en el ámbito de la economía y los negocios. Nuestro trabajo propone una versión de la racionalidad práctica dialógica-cordial que, integrando los avances de la neuroética en la perspectiva de la racionalidad discursiva, supere la racionalidad estratégica calculadora y el intuicionismo emotivista.
\end{abstract}

PALABRAS CLAVE: racionalidad práctica, neuroética, toma de decisiones, ética discursiva, responsabilidad, neuroeconomía.

\section{Neuroethics transformation of economic rationality: implications for decision making in business}

ABSTRACT: The economy and business field has traditionally been dominated by a conception of calculator, strategic and profit-maximizing individual rationality. This model of rationality has important theoretical limitations and practical consequences. In recent decades the results of neuroethics ' research seems open new ways for understanding the practical rationality. These new routes, representing a revaluation of emotional and intuitive dimension in cognitive ability and have great relevance for business and economic decision making.

The aim of this paper is to analyse the implications of a rational dialogic-friendly (cor-cordis) concept for economic and business decision-making. Our work proposes a version of the dialogic-friendly practical rationality that integrate advances in neuroethics with the discursive rationality perspective in order to overcome the strategic rationality and emotivist intuitionism.

KEY WORDS: practical rationality, neuroethics, decision making, discursive ethics, responsibility, neuroeconomics.

\section{INTRODUCCIÓN}

Vivimos porque tomamos decisiones, y vivimos de cierta manera porque tomamos decisiones de acuerdo a ciertas ideas. La preocupación por las razones

1 Este trabajo se enmarca dentro del proyecto de I+D «Proyecto de Investigación Científica y Desarrollo Tecnológico, con Referencia FFI2013-47136-C2-1-P, financiado por el Ministerio de Economía y Competitividad». 
para la acción y por criterios «correctos» para la toma de decisiones tiene una larga historia en la filosofía y en otros ámbitos. El problema es identificar los criterios para una decisión correcta y saber si podemos establecer una jerarquía entre ellos.

Un ejemplo de este «conflicto de principios» es el que reproduce Nida-Rümelin en su libro Economic rationality and practical reason ${ }^{2}$ donde cuenta el caso del sobrino que decide matar al tío para heredar su fortuna. En el diálogo entre el tío y el sobrino, el tío se ve obligado a reconocer que matarle sería una decisión "correcta», puesto que es el único medio que tiene el sobrino para hacerse rico de forma rápida y sin esfuerzo (que es lo que más desea en la vida). Este ejemplo, que Nida-Rümelin toma de R.M. Hare, nos obliga a una reflexión rigurosa sobre la racionalidad práctica en sus diversas manifestaciones y, en concreto, a la compatibilidad entre la racionalidad práctica económica y la racionalidad práctica ética.

El ámbito de la economía, los negocios y las empresas ha sido tradicionalmente un ámbito dominado por una concepción de racionalidad calculadora, estratégica y maximizadora del beneficio individual. Ese modelo de racionalidad tiene importantes limitaciones teóricas y graves consecuencias prácticas para el mundo de los negocios y para la sociedad en general como han puesto de manifiesto prestigiosos autores de economía y gestión ${ }^{3}$. También desde el ámbito de la filosofía moral ese enfoque ha sido fuertemente criticado tanto desde un análisis histórico conceptual ${ }^{4}$ como desde la reflexión crítica de la racionalidad económica ${ }^{5}$.

En las últimas décadas los resultados de las investigaciones neuroéticas parecen abrir nuevas vías en la comprensión de la racionalidad práctica ${ }^{6}$. Estas nuevas vías, que suponen una revalorización de la dimensión emocional e intuitiva en la capacidad cognitiva, tienen gran relevancia para la toma de decisiones en el ámbito de los negocios y las organizaciones. La neuroecomía y el neuromarketing, partiendo de la economía conductual y experimental, han

2 Cfr. NidA-Rümelli, J., Economic Rationality and Practical Reason, Dordrecht, Springer Verlag, 1997.

3 Cfr. Ghoshal, S., «Bad managers' theories are destroying good management practices». Academy of Management Learning and Education, 4, 2005, pp. 75-91. Cfr. Galbraith, J. K., La economia del fraude inocente, Barcelona, Crítica, 2004. Cfr. SEN, A. "Consequential evaluation and Practical Reason», The Journal of Philosophy, 97, 9, 2000, pp. 477-502. SEN, A., Rationality and Freedom, Cambridge, Harvard University Press, 2002.

4 Cfr. Conill, J., Horizontes de economía ética, Madrid, Tecnos, 2004. Conill, J., «Neuroeconomía y neuromarketing. ¿Más allá de la racionalidad maximizadora?», en Cortina, A. (edit.), Guía Comares de Neurofilofía práctica, Comares, Granada, 2012, pp. 39-64.

5 Cfr. UlRich, P., Transformation der ökonomischen Vernunft. Fortschriftsperspektiven der modernen Industriegesellschaft, Bern, Haupt Verlag, 1993.

6 Especialmente relevantes por su impacto y la contundencia de sus afirmaciones son las conclusiones de Greene y de Haidt. Cfr. Greene, J., Moral tribes. Emotion, Reason, and the Gap Between Us and Them, New York, Penguin Books, 2013. HAIDT, J., «The emotional Dog and its rational tail: A Social intuitionist approach to moral judgment», Psychological Review, Vol. 108, n 4, 2001, pp. 814-834. 
intentado entender los procesos neurobiológicos que subyacen a la toma de decisiones de inversión, consumo y gestión ${ }^{7}$. Estas investigaciones han supuesto relevantes avances en el conocimiento de cómo los agentes económicos actúan, y han cuestionado la imagen del homo economicus incorporando la dimensión emocional y de reciprocidad (homo reciprocans), pero, a nuestro juicio, siguen sin integrar plenamente la dimensión ética en estos nuevos enfoques.

El objetivo de este artículo es proponer un renovado concepto de racionalidad práctica dialógica cordial y presentar sus fortalezas para la toma de decisiones responsables en el mundo de los negocios. Nuestro trabajo propone una versión de la racionalidad práctica que, integrando los avances de la neuroética en la perspectiva de la racionalidad discursiva, supere la racionalidad estratégica calculadora y el intuicionismo emotivista.

\section{RACIONALIDAD PRÁCTICA ECONÓMICA}

La razón práctica tiene por objetivo pensar para actuar; y de la misma forma que la razón especulativa busca métodos para conocer, la razón práctica busca fundamentos para determinar la voluntad. El hecho de que el ser humano tenga capacidad para determinar su causalidad mediante la representación de reglas supone la asunción de la consciencia de libertad de la voluntad como un factum $^{8}$.

Esta capacidad de actuar de acuerdo a reglas es la clave de la razón práctica que se puede concretar en diversos tipos de usos. J. Habermas ${ }^{9}$ identifica tres usos de la razón práctica: pragmática, ética y moral ${ }^{10}$. Adela Cortina, por su parte, identifica cuatro grandes usos de la razón práctica: prudencial, utilitarista, deontologista y ético discursiva ${ }^{11}$. No es aquí el momento de presentar con detalle los matices y las discusiones teóricas que les concierne, pero es necesario tenerlas presente porque algunas de ellas están muy presente en el mundo de la economía y de los negocios, mientras que otras han sido completamente olvidadas y convendría recuperarlas.

En el ámbito económico con frecuencia se establece una distinción entre lo que es racional identificándolo con ventajoso, y racional como justificación

7 Op. Cit. Conill, J., 2012. Calvo, P. y González, E., «Neuroeconomía, ¿un saber práctico?» en García Marzá, D. y Feenstra, R. (eds), Ética y neurociencias. La aportación a la política, la economía y la educación, Castellón de la Plana, Publicacions de la Universitat Jaume I, pp. 93-116. 2013.

8 Cf. Kant, Kritik der praktischen Vernunft, Riga, 1788.

9 Cfr. Habermas, J., Erläuterungen zur Diskursethik, Shurkamp, Frankfurt am Main, 1991.

10 Para una reflexión detallada de estos usos y su vinculación con la evolución de la conciencia moral y lo que ello supone de superación del naturalismo que proponen algunas perspectivas neuroética, véase el artículo de Cesar ORTEGA «¿Naturalizar la idea de justicia? Una respuesta crítica desde la teoría moral de Jürgen Habermas» en este mismo monográfico.

11 Contina, A., Ética de la empresa, Trotta, Madrid, 1994. 
normativa. Para Nida-Rümelin ${ }^{12}$ esta distinción no es correcta. Una concepción individualista de la razón, como la que propone la interpretación estándar de la teoría de la decisión, hace depender la racionalidad de una acción de la deseabilidad personal y de las creencias subjetivas. Es decir, la racionalidad aquí se basa en los deseos personales y en la optimización individual del interés, que conduce en muchas ocasiones a la irracionalidad colectiva. Para Nida-Rümelin: «Una acción es racional si hay una buena razón para hacerla. La teoría de la racionalidad práctica es una teoría de las buenas razones para la acción» ${ }^{13}$. Y podemos identificar tres tipos diferentes de «buenas razones»: a) Razón consecuencialista que supone tomar decisiones con el objetivo de optimizar el estado subjetivo del agente o de otro sujeto afectado por el agente. Son razones que se toman considerando las consecuencias previsibles de nuestras acciones. b) Mantener o cumplir una promesa supone hacer algo sin perspectiva consecuencialista, sin pensar en lo que en el futuro pasará, sino por realizar una intención, promesa, deber o sentimiento de obligación. c) Razones de acción guiadas por principios. Estas razones como salvar a alguien de morir ahogado aunque nos suponga una pérdida material ${ }^{14}$ y un gran esfuerzo y riesgo para nuestra vida, no son consecuencialistas y son muy racionales.

El problema es que el enfoque dominante en la teoría económica solo considera «buenas razones» las razones de carácter consecuencialistas. Como reconoce McMurty ${ }^{15}$, un aspecto central no examinado del cálculo económico y su sistema de pensamiento es el pensamiento axiomático de «racionalidad» que subyace como regulador racional y que se concreta como: ser racional es maximizar consistentemente su propio interés en términos de valor-dinero.

Así pues, desde esta perspectiva económica, una decisión racional es aquella que se basa en la evaluación de consecuencias que supone que: «(...) cualquier elección de acción debe basarse en seleccionar alguna alternativa que no produce un resultado completo peor que cualquier otra alternativa disponible ${ }^{16}$. Este modelo de evaluación tiene, a juicio de Sen, dos debilidades importantes: por un lado olvida que la evaluación siempre es situacional y que la persona que toma decisiones no puede escapar de la necesidad de tomar en consideración su posición real; y, por otro lado, olvida la distinción entre los «resultados comprehensivos», y el resultado final ${ }^{17}$. Estos dos olvidos de la racionalidad económica dominante junto a una estereotipada y simplista imagen del ser humano han llevado a Sen a considerar que la economía

12 Op. Cit. NidA-RüMELIN, 1997.

13 Íbidem, p. 130.

14 Cfr. Singer, P., «Ética e intuiciones» En Cortina, A. (ed), Guía Comares de Neurofilosofía práctica, Editorial Comares, Granada, 2012 [Traducción de Manuel Sánchez Rodriguez].

15 Cfr. McMurtry, J., "Behind Global System Collapse: The Life-Blind Structure of Economic Rationality», Journal of Business Ethics, 108, 2012, pp. 49-60.

16 Cfr. SEN, A. "Consequential evaluation and Practical Reason», The Journal of Philosophy, 97, 9, 2000, pp. 477-502, p. 478.

17 Ídem, p. 491. 
es una ciencia que opera en base a una imagen de los individuos como tontos racionales ${ }^{18}$.

Como apuntábamos anteriormente, el concepto de racionalidad prevalente en economía, se ha entendido como la posibilidad de justificar cada acto en términos de autointerés. Es lo que se ha dado en llamar «teoría de la preferencia revelada», donde lo que cuenta es la elección que hacen los sujetos en base a las predicciones aproximadas sobre la conducta del agente ${ }^{19}$. Aquí hay tres puntos discutibles: el punto de vista de las consecuencias (juzgar actos solo por las consecuencias); evaluar los actos, no las normas; y las únicas consecuencias consideradas en la evaluación de los actos son aquellas que refieren al propio interés ${ }^{20}$.

Esta racionalidad práctica propone así mismo una lúgubre imagen del ser humano como un ser individualista y egoísta que según Ghoshal responde a una ideología que encuentra su fundamento en la filosofía del individualismo radi$\mathrm{cal}^{21}$. Esta ideología, junto a los procesos de profecía autocumplida, es lo que ha llevado a los problemas de irresponsabilidad que hemos experimentado en el mundo de los negocios en las últimas décadas. Por utilizar palabras de Sen, en una de sus muchas críticas al homo oeconomicus y que resume muy bien este punto: «El puro hombre económico está muy cerca de ser un idiota social» 22 .

\section{LA TRANSFORMACIÓN DIALÓGICA Y CORDIAL DE LA RACIONALIDAD ECONÓMICA}

\subsection{Transformación discursiva de la racionalidad económica}

En su Transformation der ökonomischen Vernunft $t^{23}$ y de forma más profunda en Integrative Wirtschaftsethik ${ }^{24}$ Peter Ulrich analiza la actual racionalidad (o irracionalidad) económica a la luz del paradigma de racionalidad comunicativa. Su pretensión es transformar los fundamentos normativos de la racionalidad utilitarista hoy vigentes, hacia la racionalidad comunicativa deseable, que nos lleve hacia un progreso racional de la vida práctica en las sociedades del capitalismo moderno. En sus propias palabras: «La ética económica integrativa reconoce su tarea fundamental en aclarar la relación categorial entre la razón ética y la racionalidad económica; y resolver el problema de su mediación sistemática ${ }^{25}$.

Tal y como lo plantea Ulrich, el problema económico tiene dos dimensiones: a) El problema normativo de la determinación de los criterios racionales de

18 SEN, A., "Rational Fools: A Critique of the Behavioural Foundations of Economic Theory», Philosophy and Public Affairs, vol. 6, n 4, pp. 317-344.

19 Cfr. Op. Cit. Calvo, P. y GonzÁlez, E., 2013.

20 Íbidem, p. 342

21 Op. Cit. Ghoshal, 2005.

22 Op. Cit. SEN, 1977, 336.

23 Ulrich, P., Transformation der ökonomischen Vernunft, Haupt, Bern, 1993.

24 Ulrich, P., Integrative Wirtschaftsethik, Haupt, Bern, 1997.

25 UlRich, 1997, p. 96. 
valoración de la acción económica, y b) La indagación sobre las estrategias de acción racional orientada a fines. En la primera dimensión de lo que se trata es de una valoración de los fines de la actividad económica, y en el segundo se trata de las mejores estrategias para alcanzar ese fin. Así, este problema de la racionalidad económica implica dos dimensiones de la racionalidad: la configuración de la interacción social (entendimiento racional) y el uso eficiente de los recursos (la disposición racional). El problema es que la economía parece haberse centrado solo en la dimensión estratégica para el uso eficiente de recursos.

La racionalidad económica dominante ha partido del supuesto que la acción económica se orienta a metas para la satisfacción de deseos y necesidades humanas a través de la disposición de todo tipo de bienes. Dado que no tenemos recursos para todas las necesidades y deseos se genera el conflicto que se resuelve mediante la competencia. El actor de esa competición es el homo oeconomicus que se llega a concebir como una realidad natural ${ }^{26}$ totalmente desconectada del «mundo de la vida». Pero como Ulrich pone de manifiesto, el homo oeconomicus es muy poco racional, (y poco ser humano, añadimos nosotros) por las siguientes carencias: a) Según el modelo ideal del homo oeconomicus, éste tiene información completa para tomar decisiones de acuerdo a sus deseos, pero ignora todo lo demás; b) Tiene problemas de motivación, puesto que solo busca maximizar las ganancias materiales olvidando las necesidades sociales inmateriales; c) Tiene problemas de comunicación, le falta la palabra, es un buen calculador pero es incapaz de comunicar; y d) Opera en un vacío institucional, parece que sólo se guía por sus propios fines y que no existen instituciones, prácticas sociales, o formas de control social que influyan en su comportamiento.

Desde este análisis crítico del modelo de actor económico por excelencia, Ulrich propone la reconstrucción de la racionalidad económica desde un reacoplamiento de la economía científica y el mundo de la vida. En esencia, lo que Ulrich plantea es transformar la racionalidad estratégica, calculadora, individualista y egoísta en una racionalidad socioeconómica integrada con el mundo de la vida y legitimada discursivamente.

\subsection{Transformación cordial de la racionalidad económica}

La interesante propuesta de transformación de la racionalidad económica propuesta por Ulrich, supone a nuestro juicio un importante avance respecto a la concepción de la racionalidad económica neoclásica, pero todavía tiene unas carencias que pueden ser compensadas desde una ética de la razón cordial propuesta por A. Cortina ${ }^{27}$. La ética del discurso, como sabemos, se basa en el reconocimiento recíproco de sujetos que se saben dotados de competencia co-

26 Aquí Ulrich, se refiere a RicARDo, D., On the principles of Political economy and taxation, London, 1817.

27 Cfr. Contina, A., Ética de la razón cordial, Oviedo, ediciones Novel, 2007. Cortina, A., Neuroética y neuropolítica, Madrid, Tecnos, 2011. Cortina, A. (ed.), Guía Comares de Neurofilosofía práctica, Granada, Comares, 2012. 
municativa y que se sienten obligados unos con otros más allá de la proximidad física o genética y del interés prudencial, pero sigue atendiendo de forma muy limitada a la dimensión emocional y social del ser humano. Y aquí es donde la razón cordial hace su gran aportación al recuperar la forja del carácter y la calidez humana para la ética dialógica de origen kantiano.

La ética de la razón cordial sigue tomando como punto de partida el factum de la argumentación, pero asume que el ser humano: «(...), no sólo revela una capacidad de argumentar sobre lo verdadero y lo justo, sino que cuenta también con una dimensión cordial y compasiva, sin la que no hay comunicación. ${ }^{28} \mathrm{La}$ dimensión cordial y compasiva no es ni complementaria ni secundaria respecto al discurso lógico, sino que es parte sustancial de ella en tanto en cuanto supone: «prenociones estéticas para la disponibilidad de ánimo a los conceptos de deber ${ }^{29}$. La conciencia moral, el sentimiento moral, el respeto por sí mismo (la autoestima), y el amor al prójimo son cuatro condiciones subjetivas de la receptibilidad para el concepto de deber que se condensan en el auténtico reconocimiento recíproco. Este reconocimiento crea un vínculo, una ligatio, que debe entenderse como un vínculo lógico discursivo entre los virtuales participantes en un diálogo y al mismo tiempo como un vínculo emocional entre seres humanos que se reconocen con igual dignidad.

La propuesta de la ética cordial no sólo se basa en el factum de la dimensión emocional y social del ser humano (algo que la neuroética ha destacado enérgicamente) sino también en la reflexión sobre la idea de justicia. Según la propuesta de Cortina, la voluntad y la aspiración a la justicia no pueden entenderse sin un compromiso con los vulnerables y con la realidad de los que sufren. La compasión (otro sentimiento enormemente revalorizado por la neuroética), ese sentimiento moral de dolor ante los que sufren, es el primer paso para la acción en pro de la justicia.

Esta concepción de la ética de la razón cordial se concreta en cinco grandes principios prácticos. El primero, en la más ilustre tradición kantiana, es no instrumentalizar a las personas y tratarlas siempre de acuerdo a su dignidad. El segundo, siguiendo el enfoque de capacidades, es empoderar a las personas para que puedan ser interlocutores efectivos en los discursos prácticos. El tercero se centra en la distribución equitativa de las cargas y los beneficios, teniendo como referencia intereses universalizables y la creación de una sociedad justa. El cuarto tener dialógicamente en cuenta a los afectados por las normas a la hora de tomar decisiones sobre ellos. Y, el quinto minimizar el daño en el caso de los seres sentientes no humanos y trabajar por un desarrollo sostenible (principio de responsabilidad por los seres indefensos no humanos).

Estos cinco principios son concreciones de una razón cordial que como afirma Cortina pretende recoger la esencia de la razón en todas sus dimensiones y parte de la convicción de que:

28 Cfr. Cortina, A., 2007, p. 191

29 Cfr. Cortina, A., 2007, p. 120 
«Oponer la lógica del corazón a la de la razón es costumbre tan antigua como infortunada, porque la razón es una facultad preparada para interpretar proyectos del corazón, para extenderlos en propuestas teóricamente elaboradas, pero esos proyectos racionales sólo cobran fuerza motivadora si no pierden su arraigo en el corazón» ${ }^{30}$.

\section{APORTACIONES DE LA NEUROÉTICA A LA COMPRENSIÓN DE RACIONALIDAD PRÁCTICA EN LA ECONOMÍA Y LOS NEGOCIOS}

El debate sobre el alcance de los avances de la neuroética para la filosofía moral sigue muy activo, pero parece fuera de toda duda que entender cómo elaboramos juicios morales nos puede ayudar a entender cómo tomamos decisiones morales, a comprender nuestro comportamiento y a buscar caminos más efectivos para la realización de nuestros ideales morales.

Tal y como hemos presentado en las páginas anteriores, el estereotipo ideal de la racionalidad económica ha sido el individuo autónomo, calculador y egoísta $^{31}$. Pero esta visión empezó a resquebrajarse a finales de los años 90 con las primeras aportaciones de las investigaciones neuroeconómicas de Shizgal y Conover ${ }^{32}$. Así, nos atreveríamos a identificar cuatro grandes impactos de los avances de las neurociencias a la racionalidad económica:

a) Más allá del individualismo. Un presupuesto extendido en la teoría económica dominante es que las personas somos agentes individuales que hacemos cálculos para satisfacer nuestros deseos. Y que dado que los recursos son escasos, la mejor manera de entender el comportamiento de las personas es la competición por dichos recursos. Según las conclusiones de las investigaciones en neurociencia es más bien lo opuesto. El ser humano está diseñado para la cooperación:

«Tenemos cerebros cooperativos, parece ser, porque la cooperación ofrece beneficios materiales y recursos biológicos que permite a nuestros genes hacer más copias de ellos mismos. De la suciedad evolutiva crece la flor de la bondad humana» ${ }^{33}$.

Así, aunque se reconoce que hay una competencia por los recursos escasos esa competencia no es individual, sino grupal, es decir, se compite entre grupos, pero no intragrupos. En este sentido la idea del individuo decisor racional que decide en base a sus preferencias es inexacto desde el punto de vista antropológico y evolutivo. La idea de que el ser

\footnotetext{
30 Cfr. Cortina, A., 2007, p. 126.
}

31 Para un estudio más pormenorizado de la aportación de la neuroética a la racionalidad económica puede verse: CALVO, P., «Neuro-racionalidad: heterogeniedad motivacional compromiso moral», Daimon. Revista internacional de filosofía, 59, p. 157-170. 2013.

32 Shizgal, P. y Conover, K., "On the neural computation of utility», Current directions in Psychological Science, 5 (2), pp. 37-43. 1996.

33 Cfr. Greene, 2013, p. 65. 
humano es un ser egoísta y competitivo por naturaleza parece bastante cuestionado desde las neurociencias. Según Greene ${ }^{34}$ la cooperación ha sido clave en nuestra evolución y ha generado una serie de disposiciones emocionales que ha sido clave en el surgimiento de la moralidad. Aunque no compartimos la perspectiva naturalista ${ }^{35} \mathrm{de}_{\text {Greene sí nos parece }}$ interesante resaltar la idea de que la preocupación por otros (empatía), la reciprocidad directa (gratitud, enfado y disgusto), el compromiso con las amenazas y las promesas (venganza, vergüenza y culpabilidad), la reputación, y la reciprocidad indirecta son capacidades naturales del ser humano que le predisponen para la cooperación y la generosidad antes que para la vida aislada e individual.

b) Más allá del autocontrol. Otro de los presupuestos de la racionalidad económica es que una persona va a actuar siempre tomando decisiones que le acerquen a la consecución de sus deseos. Los avances en neurociencias demuestran que tenemos menos control sobre muchas de nuestras decisiones de lo que solemos pensar; o, dicho con otras palabras, que nuestra capacidad para actuar de acuerdo a nuestros fines no es tan fuerte como pensamos. La fuerza de la voluntad y el autocontrol no es tan potente como se presupone en economía. Así, nuestra capacidad para posponer la recompensa o nuestra renuncia a hacer conductas que creemos ciertamente que no debemos hacer (como cuando no ahorramos todo lo que pensamos que deberíamos, o consumimos productos que pensamos que no deberíamos) son ejemplos de ello. El fenómeno de la akrasia es un tema que la neuroética ha vuelto a poner en el centro de la reflexión ${ }^{36}$.

c) Más allá del análisis computacional. Uno de los avances más controvertidos de la ciencias cognitivas ha sido la crítica a la racionalidad analítica more cartesiano ${ }^{37}$. Nuestros procesos cognitivos son menos lógico analíticos, y mucho más heurísticos de lo de que solíamos pensar. La heurística es: «un proceso mental que ignora parte de la información accesible y que no pretende optimizar, lo que significa que no realiza una computación de máximos y mínimos» ${ }^{38}$. Los atajos mentales, los sesgos de confirmación, las reglas de sentido común, junto con la debilidad de nuestros recuerdos cuestionan seriamente nuestra imagen de personas analíticas que se toma como base de la racionalidad

34 Cfr. Greene, 2013, pp. 60-61.

35 Más bien compartimos la crítica de Javier Gracia a Greene, cuando afirma que cae en la falacia naturalista. Veáse en este mismo número: GrAciA, J., “¿Incurre la teoría del proceso dual de Joshua Greene en falacia naturalista?» 2016.

36 Cfr. Levy, 2008. Para un estudio riguroso de este fenómeno puede verse el artículo de P. J. Teruel en este mismo número.

37 Damasio, A., Descartes 'Error. Emotion, Reason, and the human brain, Penguin books, New York, 1994.

38 Cfr. Gigerenzer, 2010, p. 2 
económica. De hecho los marcos de referencia (framing), las narrativas, y los modelos mentales orientan no solo nuestras decisiones, sino también las percepciones de la realidad. La heurística tiene dos dimensiones, una positiva que se identifica con la eficiencia cognitiva, y una negativa que se identifica con la toma de decisiones irracional ${ }^{39}$. Es esta segunda dimensión la que más está afectando a la economía. También autores como D. Kahneman ${ }^{40}$ y D. Ariely ${ }^{41}$ han demostrado que nuestra aversión a las pérdidas nos lleva en ocasiones a aceptar decisiones que no son óptimas desde un punto de vista de maximización de utilidades. Y esas decisiones son más relevantes de lo que tendemos a pensar ${ }^{42}$. Como D. Kahneman afirma: «(...) [las] preferencias intuitivas (...) violan las reglas de la elección racional consistentemente» ${ }^{43}$.

Vinculado a este punto también es importante destacar que las investigaciones han constatado que las personas somos muy torpes a la hora de procesar números. Teniendo presente esta afirmación, y considerando que la gestión tiende a reducir a números toda la realidad económico-social, pues hay un riesgo realmente alto de que tomemos malas decisiones ${ }^{44}$.

d) Más allá del egoísmo. Como ya hemos apuntado anteriormente. El modelo del homo oeconomicus como un calculador de utilidades está sólidamente criticado. Como afirma A. Sen, la idea de que el homo oeconomicus actúa sólo honestamente mientras obtiene algún beneficio económico «(...) está lejos de ser una verdad obvia y necesita confrontación con la realidad observable» ${ }^{45}$. En ese artículo Sen defiende la importancia del «compromiso» como un elemento clave que va más allá del cálculo de intereses para entender el comportamiento económico. Los resultados en neurociencias, basados en los resultados del juego del ultimátum, apuntalan esta observación al reconocer que las personas están dispuestas a perder ventajas económicas porque la decisión la consideran injusta, porque se sienten indignados, por afán de venganza, o por mantener la reputación social ${ }^{46}$. Y en la misma línea es importante

39 Cfr. Dubljevic, V.; Racine, E., «The ADC of Moral Judgment: Opening the Black Box of Moral Intuitions with Heuristics About Agents, Deeds, and Consequences», AJOB Neuroscience, 5 (4), 2014, pp. 3-20.

40 Cfr. Kahneman, D., Thinking fast and Slow, Farrar, strauss and Giroux, New York, 2011.

41 Cfr. Ariely, D., Predictably Irrational, Harper Perennial, New York, 2009.

42 Cfr. LeVY, 2008.

43 Cfr. Kahneman, op. cit. p. 10.

44 Cfr. Kahneman, 2011. Especialmente el capítulo 11 donde se explica el «efecto gancho» y como nuestra capacidad para estimar cantidades es muy imprecisa.

45 Cfr. SEN, 1977, p. 332.

46 Cfr. SAnfey, A. G. et al., "The Neural Basis of Economic Decision-Making in the Ultimatum Game», Science, vol. 300, 2003, pp. 1755-1758. SANFEY, A. «Expectations and social decision-making: biasing effects of prior knowledge on Ultimatum responses», Mind Society Journal, 8, 93-107, 2009. 
reconocer la capacidad del ser humano de juzgar intenciones de otros actores. Es decir, a las personas no sólo nos interesa el resultado que obtenemos, sino también el proceso. Así, en el experimento de Sanfey, cuando quien ofrecía unos tratos realmente injustos era el ordenador, los receptores aceptaban la cantidad propuesta en un mayor porcentaje que cuando los tratos injustos los ofrecían otras personas ${ }^{47}$.

Estas cuatro grandes cuestiones ya hace tiempo que se han tomado en consideración en la economía y han generado una nueva disciplina que se llama neuroeconomía y su derivado el neuromarketing. La neuroeconomía se ha entendido como: «el campo que, a partir de la economía conductual y de la experimental, intenta entender los procesos neurobiológicos que subyacen a la toma de decisiones ${ }^{48}$, y una de sus principales aportaciones ha sido acercarse al mundo real, superando las idealizaciones lógicas y matemáticas de la teoría económica estándar y cambiar la imagen estereotipada del ser humano. El gran objetivo de la neuroeconomía es entender las bases neurobiológicas de cómo y por qué las personas hacen juicios y toman decisiones que tienen consecuencias económicas. El objetivo final es que los resultados de esta investigación mejoren la práctica y la toma de decisiones en el derecho, la gestión, la salud pública y otros ámbitos de la vida social.

Se trata, en definitiva, de ampliar la tradicional concepción de racionalidad económica no solo para una más precisa comprensión teórica sino para mejorar la práctica económica y la toma de decisiones en los negocios. Como afirma A. Cortina:

«Lo bien cierto es que también en economía resulta imposible hacer predicciones sobre las acciones humanas o diseñar una teoría correcta de la racionalidad sin tener en cuenta otras motivaciones, independientemente de la propia autosatisfacción, como es el caso de la preocupación por el bien de los otros ${ }^{49}$.

5. IMPLICACIONES PRÁCTICAS PARA LA TOMA DE DECISIONES EN EL MUNDO EMPRESARIAL

La primera implicación de los resultados de la investigación neurocientífica para la toma de decisiones éticas es su especificidad. Las investigaciones neurológicas parecen avalar que la toma de decisiones éticas es diferente a otros procesos de toma de decisiones ${ }^{50}$.

«Los estudios ofrecen evidencias de que el conocimiento, el juicio y la conducta moral son distintos de otras formas cognitivas de procesos de toma

47 SANFEY, A., 2003, p. 1756

48 Conill, J., 2012, p. 42.

49 CORTINA, 2007, p. 75.

50 Dawn R. Elm y Tara J. Radin, «Ethical Decision making: Special or no different?» Journal of Business Ethics, ${ }^{\circ}$ 107, 2012, pp. 313-329. 
de decisiones en el sentido que la toma de decisiones éticas no solo parece ser independiente de la habilidad intelectual, sino que también activa mecanismos neuronales distintos de otros asociados con otros procesos mentales» ${ }^{51}$.

El estudio de la toma de decisiones ética en la empresa ha comenzado en muchas ocasiones por su parte negativa. Es decir, por identificar los factores que influyen en la toma de decisiones no éticas ${ }^{52}$ y para intentar responder a la pregunta de por qué las conductas y decisiones no éticas son tan frecuentes en el mundo de los negocios a pesar de los sistemas de vigilancia y control ${ }^{53}$.

\subsection{Modelos «racionalistas» de toma de decisiones en el Management}

Incluso desde los enfoques más matemáticos de la toma de decisiones como los Analytic Hierarchy Process $(\mathrm{AHP})^{54}$ o los Multi-criteria Decision Analysis $(\mathrm{MCDA})^{55}$ se reconoce que tomar una decisión no es simplemente una cuestión de seleccionar la mejor alternativa sino que implica una complejidad y multidimensionalidad de los procesos de toma de decisión que exige un esfuerzo intelectual, de reflexión, y de abstracción muy elevado y donde las mentalidades y las emociones juegan un papel central ${ }^{56}$.

Una asunción en los modelos de toma de decisiones en la dirección de empresas es que los individuos son actores con propósitos racionales que actúan de acuerdo con sus intenciones y entienden las implicaciones de sus acciones. Son modelos que han sobrevalorado las explicaciones causales y funcionales deterministas ${ }^{57}$. Estos modelos siguen un proceso causal lineal de cuatro fases: 1) reconocimiento de los asuntos morales, 2) juicio moral, 3) intento moral, y 4) conducta moral.

Este enfoque ha tomado como punto de partida la teoría de Kohlberg $^{58}$ y Rest $^{59}$ sobre la evolución del juicio moral y se han centrado en analizar cómo los factores del contexto organizacional y la intensidad moral de los asuntos in-

51 Cfr. Salvador, R. and Folger, R. G., «Business ethics and the Brain» in Business Ethics Quarterly, Vol. 19, n' 1, 2009, pp. 1-31.

52 Moore, C.; Detert, J. R.; Treviño, L. K.; Baker, V. L.; Mayer, D. M. (2012), «Why Employees do bad things: Moral Disengagement and unethical behavior», Peronnel Psicology, 65 , pp. $1-48$.

53 De Cremer, D.; Van Dick, R.; Tenbrunsel, A.; Pillutla, M.; and Murnighan, J. K. , «Understanding Ethical Behavior and Decision Making in Management: A Behavioural Business Ethics Approach», British Journal of Management, 22, 2011, pp. 1-4.

54 SaAty, Th. L., Decision Making in Complex Environment. The Analytic network Process for Dependenceand Feedback, Pittsburg, Creative Decision Foundation, 2002.

55 Wenstop, F., «Mindsets, Rationality and Emotion in Multi-criteria Decision Analysis», Journal of Multicriteria Decision Analysis, 13, 2005, pp. 161-172.

56 Cfr. SAATY, 2002. Wenstop, 2005 Op. Cit.

57 GHoshaL, S. 2005.

58 Kolhberg, L., Essays on Moral Development, Vol. I: The Philosophy of Moral Development. San Francisco, CA: Harper \& Row. 1981.

59 Rest, J. R., Moral development: advances in research and theory, Praeger, New York, 1986. 
fluyen en todas las fases de la toma de decisiones. Entre los factores del contexto organizacional que han estudiado están: la dinámica de grupos, los factores de autoridad y los procesos de socialización. Según una extensa revisión de la literatura realizada por $\mathrm{O}^{\prime}$ Fallon y Butterfield ${ }^{60}$ los factores de la organización que más influyen en la toma de decisiones ética son los códigos éticos, la cultura y clima ético, y los sistemas de recompensa y sanciones. Por su parte, entre los factores de la intensidad moral se encuentran: la magnitud de las consecuencias, el consenso social sobre una situación, la probabilidad de los efectos, la inmediatez temporal, la proximidad física, y la concentración de los efectos. Para Jones ${ }^{61}$ la clave de la toma de decisión ética está en la percepción de la intensidad (gravedad) del asunto ético en cuestión. Desde los mismos presupuestos de racionalidad lineal-causal se presenta el modelo de Trevino ${ }^{62}$, quien concluye que la toma de decisiones éticas es el resultado de la interacción entre el juicio moral de los agentes y los factores situacionales.

Estos modelos parten del supuesto que la capacidad del juicio moral y razonamiento moral funcionan de forma analítica, secuencial y que la clave del comportamiento ético está en los factores «externos» al proceso de juicio moral.

\subsection{Modelos «intuicionistas» de toma de decisiones en el Management}

Basados en los avances de la psicología cognitiva y las neurociencias se han propuesto dos interesantes modelos que cuestionan y pretenden superar los modelos racionalistas presentados anteriormente: el modelo de "creación de sentido intuitivo» (Sensmaking-intuition model-SIM), presentado por Sonenshein ${ }^{63}$, y el modelo de creación de Sentido de Thiel et. Ali ${ }^{64}$.

Sonenshein comienza haciendo una revisión crítica de los enfoques racionalistas de Jones ${ }^{65}$ y Trevino ${ }^{66}$. A su juicio, estos enfoques racionalistas tienen cuatro importantes limitaciones: 1) Incertidumbre y equivocidad. En los dilemas éticos, normalmente, se presentan diferentes interpretaciones simultáneas y se da una grave falta información y de precisión. Las personas solemos tener tener una ilusión positiva sobre las consecuencias de nuestras decisiones y solemos estimar por lo bajo los impactos negativos. También somos muy imprecisos en

${ }^{60}$ Cfr. O’Fallon, M. J. and Butterfield, K. D., «A review of the empirical ethical decisionmaking literature: 1996-2003», Journal of Business Ethics, 59, 2005, pp. 375-413.

${ }^{61}$ Cfr. Jones, T. M., «Ethical decision making by individuals in organizations: an issuecontingent model», Academy of Management Review, vol. 16, n 2, 1991, pp. 366-395.

${ }^{62}$ Cfr. Trevino, L., «Ethical Decision Making in Organizations: A Person-Situation Interactionist Model», The Academy of Management Review, Vol 11, n 3, 1986, pp. 601-617.

63 Cfr. Sonenshein, 2007, op. cit.

64 Cfr. Thiel, Ch. E., Bagdasarov, Z., Harkrider, L., Johnson, J. F. and Mumford, M. D., «Leader ethical decision making in organizations: Strategies for snesemaking», Journal of Business Ethics, $\mathrm{n}^{\circ}$ 107, 2012, pp. 49-64.

65 Cfr. Jones, T. M., "Ethical decision making by individuals in organizations: An issuecontingent model», Academy of Management Review, Vol. 16. 1991, pp. 366-395.

66 Cfr. Trevino, 1986. 
la definición del problema ético, y hay una gran variedad a la hora de definir una situación como ética o no ética. 2) Deliberación y racionalización. El modelo racionalista sugiere que la deliberación y la racionalización son pasos previos para la toma de conciencia moral y, por tanto la respuesta conductual debe ser consistente con el punto de vista moral. Pero, como hemos visto, los recientes avances en psicología cognitiva nos demuestran que los individuos se implican en ese tipo de deliberación y racionalizaciones previas mucho menos de lo que tendemos a creer ${ }^{67}$. 3) Objetividad de las situaciones. Los enfoques racionalistas sugieren que el proceso comienza con un problema y que los sujetos reaccionan a los dilemas éticos que ya existían. Pero las investigaciones demuestran que los individuos desarrollan interpretaciones subjetivas de los temas que van más allá de las características objetivas. 4) El razonamiento y juicio moral. Basándose en $\mathrm{Haidt}^{68}$, Sonenshein considera que no es cierto que el juicio moral sea resultado del razonamiento moral. Sino que más bien parece que respondemos a los asuntos morales intuitivamente y que luego los justificamos.

Para superar estas limitaciones, Sonenshein propone su modelo de creación de sentido intuitivo (SIM) que se compone de tres niveles: construcción del asunto, juicio intuitivo, y explicación y justificación. En esencia, este modelo afirma que los individuos construyen los asuntos desde los estímulos sociales en entornos de incertidumbre y equivocidad, y muy influidos por sus expectativas $^{69}$ y motivaciones. La clave está en cómo los individuos crean sentido elaborando historias desde un conjunto de estímulos del contexto. Las personas creamos historias para dar sentido a los eventos del contexto, sin tener en cuenta si son precisas o no ${ }^{70}$, y pensamos con metáforas más que con datos ${ }^{71}$. En esta línea, la heurística difusa propuesta por Gerd Gigerenzer ${ }^{72}$ afirma que ignorar cierta información es la mejor estrategia de toma de decisiones en muchos ámbitos, y que los humanos utilizamos la heurística con gran éxito ${ }^{73}$.

El segundo paso, es el juicio intuitivo. Las investigaciones han demostrado que los individuos raramente cambian su opinión de su respuesta inicial, incluso cuando se les presentan nuevas evidencias ${ }^{74}$. Las investigaciones neurológicas apuntan que los procesos cognitivos deliberativos se utilizan fundamentalmente para racionalizar intuiciones más que para realizar juicios activos. El modelo de creación de sentido intuitivo asume que el juicio es instantáneo y que la base del juicio moral está en la reacción afectiva del sujeto al asunto. Es decir,

67 Cfr. Damasio, 1994. A. Damasio: Looking for Spinoza. Joy, sorrow and the feeling brain, Orlando, Harcourt, 2003; Haidt, 2001; Ariely, 2008; Kahneman, 2011.

68 Cfr. HaIDT, 2001.

${ }_{69}$ Especialmente relevante en este punto es el papel de las expectativas creadas como ha puesto de manifiesto Sanfey. Cfr. Sanfey 2009.

70 Cfr. Kahneman, 2011.

71 Cfr. LakKof, G. and Johnson, M., Metaphors we live by, Chicago, University of Chicago Press, 1980.

72 Cfr. Gigerenzer, 2010.

73 Cfr. Dubljevic and Racine, 2014.

74 Cfr. ARIELY, 2008. 
una vez catalogado una situación como relevante moralmente, generamos una reacción automática basada en la afectividad; una afectividad que viene determinada por las experiencias individuales y por las presiones sociales. Este momento es el que se utiliza el sistema cognitivo reflectivo ${ }^{75}$.

Y finalmente, el tercer paso, es la explicación y justificación. Los individuos justifican y racionalizan sus intuiciones. Las personas emplean las herramientas del análisis racional después de los hechos. Describimos nuestras decisiones en términos racionalistas y nos esforzamos por darle coherencia a la historia (nuestra decisión o acción) antes que ser fieles a los hechos. Como afirma Kahneman: «[...] cuando la gente cree que una conclusión es cierta, es más probable creer los argumentos que parecen apoyarla, incluso cuando esos argumentos sean poco fiables ${ }^{76}$. Es la coherencia lo que importa antes que la fiabilidad o precisión de la información.

El modelo propuesto por Thiel et al. parte de la afirmación de que el enfoque tradicional es inadecuado para entender cómo los directivos toman decisiones en condiciones de incertidumbre y equivocidad, y que el modelo de creación de sentido es más adecuado. La creación de sentido es un proceso cognitivo mediante el cual se elaboran modelos mentales que superan los déficits de información y las evaluaciones imprecisas y contribuyen a generar unas evaluaciones más eficaces. La propuesta de Thiel et. al. para fomentar la toma de decisiones responsable pasa por desarrollar estrategias cognitivas de creación de sentido: regulación emocional, auto-reflexión, pronóstico, e integración de la información.

Así pues, podemos concluir que los modelos de toma de decisiones inspirados en las teorías de la elección racional son un marco inadecuado para entender cómo los directivos responden a los dilemas éticos en condiciones de incertidumbre y confusión, y que los modelos de creación de sentido responden mejor a la realidad. En ambos modelos el aspecto central es, de nuevo, la valoración de las emociones y la crítica al modelo de decisor-máquina presentado en los enfoques tradicionales. Como reconoce Sanfey:

«Los modelos económicos estándar de toma de decisiones humanas (como la teoría de la utilidad) normalmente han minimizado o ignorado la influencia de las emociones en conducta de la toma de decisiones de la gente, idealizando al decisor como una máquina cognitiva perfectamente racional» ${ }^{77}$.

\section{Conclusiones: la Ética cordial en la toma de decisiones en el Management}

Los modelos intuitivos y de creación de sentido presentados anteriormente han realizado críticas superadoras a los modelos racionalistas de toma de decisiones en economía, pero han tenido el riesgo de caer en un intuicionismo emotivista que olvide la argumentación racional. A nuestro juicio, una propuesta

75 KaHneman, 2011.

76 Ibid. p. 45.

77 Cfr. Sanfey, 2003, p. 1755. 
más equilibrada, fundamentada y constructiva es la propuesta de ética de la razón cordial que puede ser un buen marco para desarrollar orientaciones específicas para la toma de decisiones ética en la gestión de organizaciones y en los negocios.

La pregunta clásica kantiana ¿qué debo hacer? No puede ser respondida ni desde el estudio del funcionamiento del cerebro, ni desde la apelación a los sentimientos. Aunque también hay que reconocer que los avances recientes de la neuroética y la comprensión de nuestros sentimientos nos pueden ayudar a tomar decisiones más justas. Pero hay que insistir en que las emociones no son fuente de legitimidad moral $^{78}$.

Como hemos presentado anteriormente, la concepción de la racionalidad cordial se concreta en cinco grandes principios prácticos: no instrumentalizar a las personas; empoderar a los participantes en el discurso; distribuir equitativamente cargas y beneficios; contar con los afectados; y minimizar el daño de los seres sentientes. Es necesario seguir investigando las implicaciones concretas del desarrollo de estos principios para la toma de decisiones responsable en la gestión de empresas y los negocios, pero aquí ya nos atrevemos a proponer tres líneas de actuación.

La «re-personalización» de la toma de decisiones. En el mundo de las empresas hay una tendencia a la estandarización positivista (a buscar indicadores y medirlo todo) y a la complejidad dinámica (establece relaciones complejas donde es difícil establecer líneas causales y donde todo cambia permanentemente). Esto crea unos escenarios de decisión que transmiten la sensación de ser dilemas morales impersonales. De entrada, este tipo de gestión basado en lo anónimo y numérico tiene sus ventajas de eficiencia y flexibilidad, pero también tiene sus riesgos al favorecer el anonimato y la irresponsabilidad. Y ante decisiones difíciles y con consecuencias negativas (trasladar una factoría a otro país, despedir a trabajadores, invertir en unas líneas de negocio o en otros) los decisores sienten que se encuentran ante dilemas morales impersonales, lo que les supone un desapego emocional y un mayor riesgo de bloqueo moral.

Fomento de las capacidades para el diálogo. La toma de decisiones responsable es producto de una reflexión y deliberación racional. En esa deliberación es cuestión inexcusable contar con los afectados, pero éste "contar con» debe ir mucho más allá de la participación nominal presencial, y de la información a los afectados. Se requiere un sistema que fomente la participación real y efectiva; y, sobre todo, se requiere personas con oportunidades reales y capacidades para participar. La ética de la razón cordial implica el empoderamiento de las personas para poder participar en los diálogos sobre las decisiones que les afectan. Así, una toma de decisiones responsable exige que se considere a los actores de la actividad económica no sólo como receptores, subordinados o competidores sino como interlocutores válidos. Esta exigencia se concreta en:

78 Ir a un funeral puede generar emociones realmente desagradables, pero eso no lo convierte en una acción inmoral, sino todo lo contrario. Cfr. DublJEvic and Racin, 2014. 
el acceso a la información por parte de los afectados, la asignación de recursos materiales y temporales para que las personas puedan participar efectivamente, y el desarrollo constante de procesos de participación donde se generen procesos de aprendizaje y de reflexión más allá de la negociación estratégica.

Integración emocional. Como ya hemos afirmado varias veces a lo largo de este artículo, las neurociencias: «(...) han venido a avalar, el de que las emociones tienen un gran peso en la formulación de juicios morales y en la vida moral ${ }^{79}$, pero también hemos añadido que las emociones no justifican decisiones morales. En el ámbito de la toma de decisiones empresariales y económicas, hay que integrar la autorreflexión sobre las propias emociones por dos razones fundamentales: la primera es porque están más presentes de lo que tendemos a pensar y nos influyen mucho más de lo que somos conscientes; y la segunda es porque es esencial desarrollar la empatía, la compasión ante el dolor ajeno, la indignación ante la injusticia, o la vergüenza ante las malas acciones, si queremos tomar decisiones auténticamente humanas y no queremos convertirnos en Phineas P. Gage ${ }^{80}$ modernos.

\section{BibLIOGRAFÍA}

Ariely, D., Predictably Irrational, Harper Perennial, New York, 2009.

Bonini, N. Ranyard, R. Mittone, L., "Special issue on «cognition and emotion in economic decision making». Introductory article: explaining economic decisions», Mind and Society, 8, 2009, pp. 1-6.

Bucciarelli, M., Khemlani, S.; Johnson-Lair, P. N., «The psychology of moral reasoning», Judgement and Decision Making, Vol. 3, n 2. 2008, pp. 121-139.

Callard, A., "Practical Reason», in Lepore, E. And Ludwig, K. (ed.), A Companion to Donald Davidson, Somerset, John Willey and Sons, 2013, pp. 32-47.

Calvo, P., «Neuro-racionalidad: heterogeniedad motivacional compromiso moral», Daimos. Revista internacional de filosofía, 59, 2013, pp. 157-170.

Calvo, P. y González, E., «Neuroeconomía, ¿un saber práctico?» en García Marzá, D. y FEenstra, R. (edts), Ética y neurociencias. La aportación a la política, la economía y la educación, Castellón de la Plana, Publicacions de la Universitat Jaume I, pp. 93-116. 2013.

Conill, J., Horizontes de economía ética, Madrid, Tecnos, 2004.

Conill, J., «Neuroeconomía y neuromarketing. ¿Más allá de la racionalidad maximizadora?», en Cortina, A. (edit.), Guía Comares de Neurofilofía práctica, Comares, Granada, 2012, pp. 39-64.

Cortina, A., Ética de la empresa, Trotta, Madrid, 1994.

Cortina, A., Ética de la razón cordial, Oviedo, ediciones Novel, 2007.

Cortina, A., Neuroética y neuropolítica, Madrid, Tecnos, 2011.

Cortina, A. (ed.), Guía Comares de Neurofilosofía práctica, Granada, Comares, 2012.

79 Cfr. Cortina, A. 2011 , p. 230.

80 Cfr. Damasio, 1994. 
Damasio, A., Descartes'Error. Emotion, Reason, and the human brain, Penguin books, New York, 1994.

Damasio, A., Looking for Spinoza. Joy, sorrow and the feeling brain, Orlando, Harcourt, 2003.

Dawn R. Elm y TARA J. Radin, «Ethical Decision making: Special or no different?» Journal of Business Ethics, $\mathrm{n}^{\circ}$ 107, 2012, pp. 313-329.

De Cremer, D. et. al., "Understanding Ethical Behavior and Decision Making in Management: a behavioural Business Ethics approach», British Journal of Management, Vol. 22, 2011, pp. 1-4.

DublJeVic, V., Racine, E., «The ADC of Moral Judgment: Opening the Black Box of Moral Intuitions With Heuristics About Agents, Deeds, and Consequences», AJOB Neuroscience, 5 (4), 2014, pp. 3-20.

Gigerenzer, G., «Moral Satisficing: Rethinking Moral Behavior as Bounded Rationality», Topics in cognitive Science, 2, 2010, pp. 528-554.

GноsнаL, S., "Bad managers' theories are destroying good management practices». Academy of Management Learning and Education, 4, 2005, pp. 75-91.

Greene, J., "Del "es" neuronal al "debe" moral». En Cortina, A. (edit), Guía Comares de Neurofilosofía práctica, Editorial Comares, Granada, 2012. [Traducción: Francisco Javier López Frías].

Greene, J., Moral tribes. Emotion, Reason, and the Gap Between Us and Them, New York, Penguin Books, 2013.

HABERMAS, J., Erläuterungen zur Diskursethik, Shurkamp, Frankfurt am Main, 1991.

HAIDT, J., «The emotional Dog and its rational tail: A Social intuitionist approach to moral judgment», Psychological Review, Vol. 108, n 4, 2001, pp. 814-834.

JoNES, T. M., «Ethical decision making by individuals in organizations: An issue-contingent model», Academy of Management Review, Vol. 16. 1991, pp. 366-395.

Kahane, G.; Wiech, K.; Shackel, N.; Farias, M.; Savulescu, J. And Tracey, I., «The neural basis of intuitive and counterintuitive moral judgement», SCAN, 7, 2012, pp. 393402.

Kahneman, D., Thinking fast and Slow, Farrar, strauss and Giroux, New York, 2011.

KANT, 1788, Kritik der praktischen Vernuntf. Riga.

Kolmberg, L., Essays on Moral Development, Vol. I: The Philosophy of Moral Development. San Francisco, CA: Harper \& Row. 1981.

Lakkof, G. and Johnson, M., Metaphors we live by, Chicago, University of Chicago Press, 1980.

Levin, I. P. et. al., «Studying Decision Processes through Behavioral and Neuroscience Analyses of Framing Effects», in Wilhelms, E. A. And Reyna, V. F. (2015), Neuroeconomics, judgement, and decision making, New York, Psychology Press, 2015, pp. $131-156$.

Levy, N., «Introducing neuroethics», Neuroethics, 1, 2008, pp. 1-8.

Lozano, J. F., «Toma de decisiones responsable en la gestión de empresas: la aportación de la neuroética». Daimon. Revista internacional de Filosofía, № 59, 2013, pp. 183- 196.

McMurtry, J., «Behind Global System Collapse: The Life-Blind Structure of Economic Rationality», Journal of Business Ethics, 108, 2012, pp. 49-60.

Moore, C.; Detert, J. R.; Treviño, L. K.; Baker, V. L.; Mayer, D. M. (2012), «Why Employees do bad things: Moral Disengagement and unethical behavior», Peronnel Psicology, 65: 1-48.

Nida-Rümelin, J., Economic Rationality and Practical Reason, Dordrecht, Springer Verlag. 1997. 
O'Fallon, M. J. and Butterfield, K. D., «A Review of the empirical ethical decision Making literature: 1996-2003». Journal of Business Ethics, 59, 2005, pp. 375-413.

Rest, J. R., Moral development: advances in research and theory, Praeger, New York, 1986.

Ricardo, D., On the principles of Political economy and taxation, London, 1817.

Rubaltelli, E.; Lotto, L.; Ritov, I.; Rumiati, R., «Moral investing: Psychological motivations and implications», Judgement and Decision Making, vol. 10, 2015, pp. 64-75.

SAaty, Th. L., Decision Making in Complex Environment. The Analytic network Process for Dependenceand Feedback, Pittsburg, Creative Decision Foundation, 2002.

Salvador, R. and Folger, R. G., «Business ethics and the Brain» in Business Ethics Quarterly, Vol. 19, n 1, 2009, pp. 1-31.

Sanfey, A. G., James K. Rilling, J. K., Jessica A. Aronson, J. A., Nystrom, L. E., Jonathan D. Cohen, J. D., «The Neural Basis of Economic Decision-Making in the Ultimatum Game», Science, vol. 300, 2003, pp. 1755-1758.

SANFEY, A., «Expectations and social decision-making: biasing effects of prior knowledge on Ultimatum responses», Mind Society Journal, 8, pp. 93-107, 2009.

SEN, A., «Rational Fools: A Critique of the Behavioural Foundations of Economic Theory», Philosophy and Public Affairs, vol. 6, no 4, 1977, pp. 317-344.

SEN, A., "Consequential evaluation and Practical Reason», The Journal of Philosophy, 97, 9, 2000, pp. 477-502.

Sen, A., Rationality and Freedom, Cambridge, Harvard University Press. 2002.

Shizgal, P. y Conover, K., "On the neural computation of utility», Current directions in Psychological Science, 5 (2), pp. 37-43. 1996.

Singer, P., «Ética e intuiciones» En Cortina, A. (edit), Guía Comares de Neurofilosofía práctica, Editorial Comares, Granada. 2012 [Traducción de Manuel Sánchez Rodriguez].

Singer, P., Famine, Affluence and Morality, Oxford, Oxford University Press, 2016.

SonENSHEIN, S., "The role of construction, intuition, and justification in responding to ethical issues at work: the sensemaking —intuition model», Academy of Management Review, Vol. 32, n 4, 2007, pp. 1022-1040.

Thiel, Ch. E.; Bagdasarov, Z.; Harkrider, L. Johnson, J. Mumford, M. D., «Leader ethical decision making in organizations: Strategies for sensemaking», Journal of Business Ethics, 107, 2012, pp. 49-64.

Trevino, L., «Ethical Decision Making in Organizations: A Person-Situation Interactionist Model», The Academy of Management Review, Vol 11, n 3, 1986, pp. 601-617.

UlRICH, P., Transformation der ökonomischen Vernunft. Fortschriftsperspektiven der modernen Industriegesellschaft, Bern, Haupt Verlag, 1993.

Ulrich, P., Integrative Wirtschaftsethik, Bern, Haupt Verlag, 1997.

WeItZner, D. And DARroch, J., «The limits of strategic rationality: ethics, Enterprise risk management, and governance». Journal of Business Ethics, 92, 2010, pp. 361-372.

Woiceshyn, J., «A model for Ethical Decision making in business: Reasoning, intuition, and rational moral principles» Journal of business Ethics, $\mathrm{n}^{\circ}$ 104, 2011, pp. 311-323.

Instituto de Gestión de la Innovación y del Conocimiento

José-FÉlix Lozano

INGENIO (CSIC-UPV)

Dpto. de Proyectos de Ingeniería

jlozan@dpi.upv.es

Universidad Politécnica de Valencia

[Artículo aprobado para publicación en octubre de 2016]. 
